\title{
RADIO DETECTIONS OF COMETARY MOLECULAR TRANSITIONS: A REVIEW
}

\author{
L. E. Snyder
}

\section{IIVTRODUCTION}

The various radio searches for cometary molecules finally came to fruition with the radio detections of $\mathrm{CH}$, $\mathrm{OH}$, $\mathrm{HCN}$ and $\mathrm{CH}_{3} \mathrm{CN}$ and several unidentified species in Comet Kohoutek (1973f) and the detection of $\mathrm{H}_{2} \mathrm{O}$ emission from Comet Bradfield (1974b) As a result of these detections we have learned something about why past radio searches of other comets for molecules were less than successful. Also, it is now possible to identify and discuss some of the similarities and differences between cometary nolecules anü interstellar molecules, particularly with regard to the excitation and chemistry Finally, given the observed projected densities and resulting gas production rates, the feasibility of future radio molecular observations of comets can be discusseà.

\section{THE IDEIVTIFIED RADIO MOLECULES}

All of the identified radio molecules have been found in two comets, Kohoutek (1973f) and Bradfield (1974b), and are listed in column 1 of taple 1. Column 2 gives the quantum numbers of each detected transition and the corresponding rest frequency is in column 3 Column 4 iists the antenna temperature for each line and column 5 shows whether the radio line was detected in enission (E) or absorption (A) The antenna half-power beamwidth (HPBW) is in column 6 and tine reported cate of the observing period is in colum 7 Column 8 has the approximate ratio of the heliocentric distance $\mathrm{R}$ to the geocentric distance $\Delta$ for the period of observation. Column 9 gives the calculated projected density $N$ (molecular density per $\mathrm{cm}^{-2}$ 


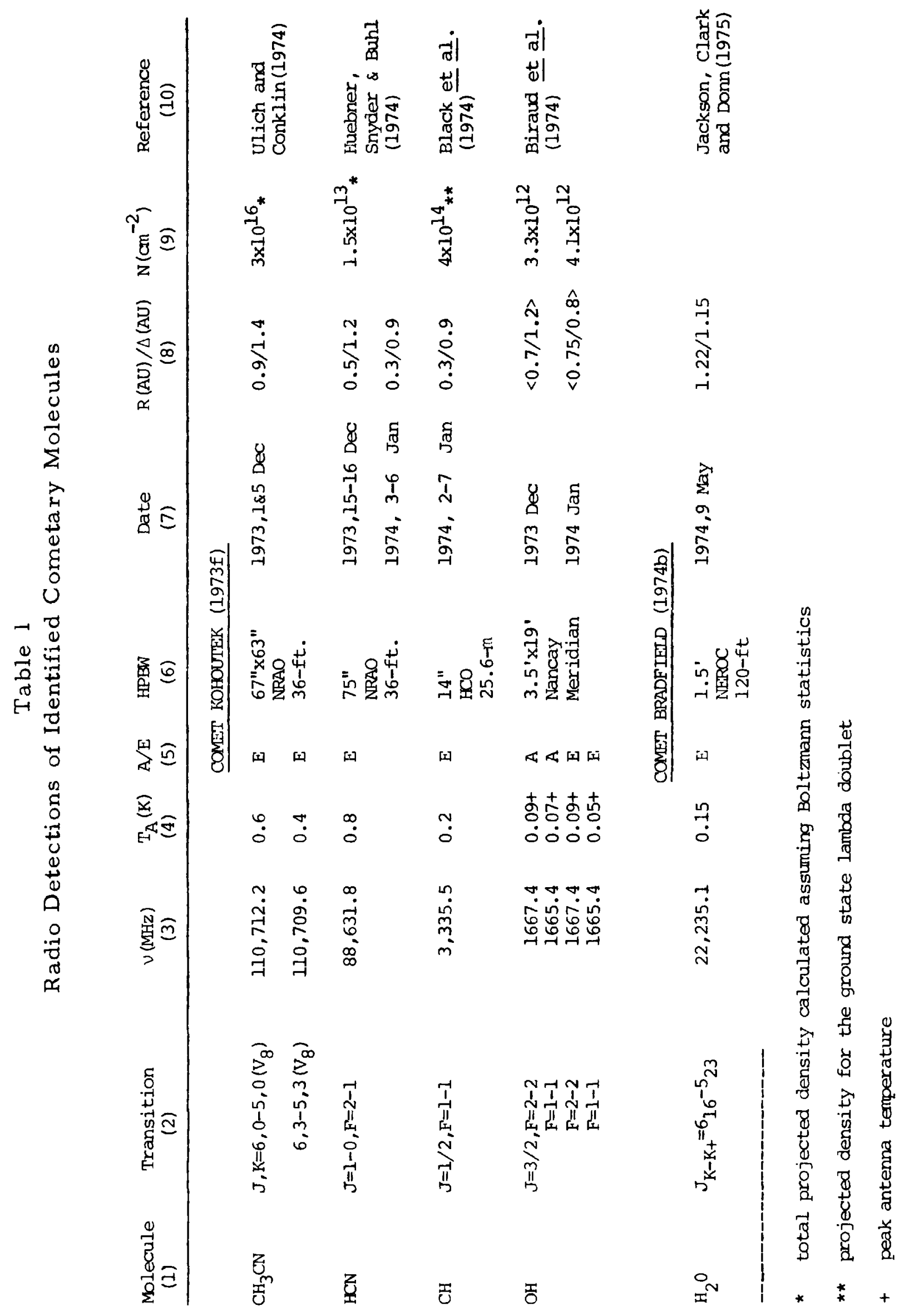


integrated along the line-of-sight) and the detection references are listed in column 10 .

Several interesting points are shown by table 1. Three of the cometary molecules were detected in the millimeter wavelength region $\left(\mathrm{CH}_{3} \mathrm{CN}\right.$ at $2.7 \mathrm{~mm}$, $\mathrm{HCN}$ at $3.4 \mathrm{~mm}$ and $\mathrm{H}_{2} \mathrm{O}$ at $13.5 \mathrm{~mm}$ ) while the remaining two were detected in the older, more developed centimeter wavelength region $(\mathrm{CH}$ at $9 \mathrm{~cm}$ and $\mathrm{OH}$ at $18 \mathrm{~cm}$ ) of the radio spectrum. Thus the millimeter wavelength region is proving to be very useful for the detection and study of cometary molecules. Given the noise limitations of currently available millimeter and centimeter radio receivers, all of the detected cometary radio lines had antenna temperatures $\mathrm{T}_{\mathrm{A}}$ (see colum 4 ) which were close to the detection limit and were weak when compared with most of the current interstellar molecular observations. This suggests that for future comet searches successful molecular detections will require a projected density in excess of about $10^{12}$ molecules per $\mathrm{cm}^{-2}$ averaged over the half-power beamwidth. Of course if the antenna beam is larger than the cometary molecular cloud, the projected density required for detection in the optically thin case will increase in proportion to the square of the ratio of the beam diameter to the molecular cloud diameter. Thus the typically smaller beamwidths in the millimeter spectral region (see colum 6) considerably reduce the beam filling factor and allow the detection of polyatomic cometary molecules which have low projected densities. Successful radio molecular observations of Kohoutek were made for $R$ less than $I$ AU (see colum 8), when the molecular production rate was high, and for $\Delta$ no greater than about $1.4 \mathrm{AU}$ so beam dilution was not excessive for the molecular species of interest.

The detections of $\mathrm{CH}_{3} \mathrm{CN}$ (Ulich and Conklin 1974), HCN (Huebner, Snyder and Bubl 1974) and $\mathrm{H}_{2} \mathrm{O}$ (Jackson, Clark and Donn 1975) were the first radio 
observations of expected parent or mother molecules in comets (Potter anā Del Duca 1974; Huebner 1970) but because of the possibility of nonequilibrium excitation, the projected densities and resulting production rates for these molecules must be interpreted with caution. For example, $\mathrm{CH}_{3} \mathrm{CN}$ was detected and observed with good signal-to-noise only in the vibrationally excited $V_{8}=1$ state which is approximately 500 K above the zero-point energy. It should be noted that vibrationally excited $\mathrm{CH}_{3} \mathrm{CN}$ never has been detected in the galactic molecular clouds, the only remotely comparable microwave transitions observed in galactic sources are the emission lines from rotational transitions of the vibrationally excited SiO maser (Snyder and Buhl 1974; Davis et al. 1974, Thaddeus et al. 1974; Buhl et al. 1974) which originate in circumstellar envelopes of late-type stars. Approximately two weeks after the $\mathrm{CH}_{3} \mathrm{CN} \mathrm{V} \mathrm{V}_{8}=1, \mathrm{~J}=6-5$ transitions were observed by Ulich and Conklin (1974), Buhl, Huebner and Snyder (1975) searched for both $V_{8}=1, J=5-4$ and ground vibrational state $\mathrm{J}=5-4$ lines of $\mathrm{CH}_{3} \mathrm{CN}$. The detected $\mathrm{CH}_{3} \mathrm{CN}$ lines were strong enough to confirm the Ulich and Conklin (1974) detection but the signal-to-noise ratio was so low that the new data were not sufficient to serve as an independent identification of cometary $\mathrm{CH}_{3} \mathrm{CN}$ (see Figure 2 in Buhl et al. 1975). The ground state lines did not have the intensities which would be expected from a Boltzmann distribution. Hence both the high excitation temperature required by $V_{8}=1$ and the transient nature of the signal strength suggest that the $\mathrm{CH}_{3} \mathrm{ON} \mathrm{V} \mathrm{V}_{8}=1$ excitation was time-varying. Therefore the projected density (colum 9 , table 1 ) and production rate of $\sim 10^{30}$ obtained by assuming a Boltzmann distribution over vibrational states of $\mathrm{CH}_{3} \mathrm{CN}$ probably are too large and a more meaningful production rate is $\sim 10^{27}-10^{28} \mathrm{~s}^{-1}$ obtained from the weak ground state signal (Buhl et al. 1975). Evidence of time-varying emission also was found for HCN. The data of Huebner 
et $\underline{\text { ai. }}$ (1974) show multiple HCN Doppler components. A component with zero shift showed increases as well as decreases in the daily average while other components appeared and disappeared during the observations. Because there was no observed evidence for nonequilibrium excitation of ifiv and because the observations lacked spatial resolution, the calculated production rates of $1.2 \times 10^{28} \mathrm{~s}^{-1}$ (for $\mathrm{r}_{\mathrm{O}}=10^{4} \mathrm{~km}$ ) to $3 \times 10^{27} \mathrm{~s}^{-1}$ (for $\mathrm{r}_{\mathrm{O}}=3 \times 10^{5} \mathrm{~km}$ ) are based on two assumptions: a Boltzmann distrivution and isotropic outstreaming of the gas (Snyder, Huebner and Buil 1975) The difficulties of calculating an $\mathrm{H}_{2} \mathrm{O}$ production rate from the radio observations of Comet Bradfield (Jackson et al. 1975) are due to both the high excitation $\left(447 \mathrm{~cm}^{-1}\right.$ ) required to populate the $6_{16}$ level and the relatively long radiative lifetime for the $6_{16}-5_{23}$ transition. As a result, $\mathrm{H}_{2} \mathrm{O}$ production rates found from applying Boltzmann statistics to radio observations tend to give numbers that are too high and a more realistic production rate probably is around $10^{28}$ (see table 1 of Jackson et al. (1975))

Radio observations of cometary OH promise to be useful for studying radiative pumping models of $\mathrm{OH}$ and hence may be directly applicable to current research on galactic OH source excitation.' In 1973 December, Biraud et al. (1974) detected $\mathrm{UH}$ absorption in Comet Kohoutek at 1665 and $1667 \mathrm{MHz}$. Turner (1974) used the NRAO $140 \mathrm{ft}$. telescope to confirm their observations while the comet was still approacining perihelion. After perihelion, however, Biraud et al. (1974) found that the $\mathrm{OH}$ reappeared in emission and that ultraviolet pumping of the ground state lambda doublet could roughly account for the observed $\mathrm{OH}$ intensities as a function of time 


\section{THE UNIDENTIFIED RADIO MOLECUIES}

Two groups of radio observers found unidentified lines while searching Comet Kohoutek for other molecules. Giguere and Clark (1975) were using the NRAO 140-ft. telescope to search for the $8190 \mathrm{MHz}$ line of $\mathrm{OH}$ when a weak unidentified emission feature at $8188.82 \mathrm{MHz}$ was detected. J.K.G. Watson (1974) has suggested that this line may be one component of the $6_{16}-5_{23}$ rotational transition of the $\mathrm{NH}_{2}$ radical. Buhl, Huebner and Snyder (1975) were using the NRAO 36-ft. telescope to search for vibrationally excited SiO when fairly strong unidentified lines at $86,247.1$ and 89,010.5 $\mathrm{MHz}$ were found. They present arguments that the 86,247 line can not be assigned to nearby transitions of ethanol, acetone or vibrationally excited silicon monoxide and suggest that one or both lines may belong to unstable species which are decay products of more complex molecules.

\section{NEGATIVE RESULTS}

In recent years numerous cometary searches have been conducted at several radio observatories but not all of the negative results have been formally reported. The reported negative results (which I am aware of at present) are ordered by frequency in table 2 Column 1 lists each molecule and indicates when a vibrationally excited state was sought, column 2 gives rotational quantum numbers and colum 3 the corresponding rest frequency Column 4 lists the upper limit reached for the peak-to-peak antenna temperature $\mathrm{T}_{\mathrm{A}}$ except when noted otherwise; column 5 , the radio telescope used, colum 6 the half-power beamwidth (HPBW) in arc minutes; and column 7, the reference.

It should be noted from columns 4 and 5 that very low antenna temperature limits were reached for $\mathrm{H}_{2} \mathrm{O}, \mathrm{H}_{2} \mathrm{CO}, \mathrm{NH}_{3}$ and $\mathrm{CH}_{2}(\mathrm{CN})_{2}$ using favorable 
Table 2

Reported Negative Results

\begin{tabular}{|c|c|c|c|c|c|c|c|}
\hline $\begin{array}{l}\text { Molecule } \\
\text { (1) }\end{array}$ & $\begin{array}{c}\text { Transition } \\
\text { (2) }\end{array}$ & $\begin{array}{c}v(\mathrm{MHz}) \\
(3)\end{array}$ & $\begin{array}{l}T_{A}(K) \\
(4)\end{array}$ & Tele & $\begin{array}{l}\text { escope } \\
\text { (5) }\end{array}$ & $\begin{array}{l}\text { HPBW } \\
(6)\end{array}$ & $\begin{array}{c}\text { Reference } \\
\text { (7) }\end{array}$ \\
\hline \multicolumn{8}{|c|}{ COMET BENNETT (1969i) } \\
\hline $\mathrm{H}_{2} \mathrm{CO}$ & ${ }^{1} 11^{-1} 10$ & $4,829.7$ & 0.30 & NRAO & $140-\mathrm{ft}$ & 6.6 & Huebner and Snyder (1970) \\
\hline $\mathrm{H}_{2} \mathrm{O}$ & ${ }^{6} 16^{-5} 23$ & $22,235.1$ & 2.5 & $\mathrm{NRL} 8$ & $85-f t$ & 2.3 & Clark et al. (1971) \\
\hline \multicolumn{8}{|c|}{ COMET KOHOUTEK (1973f) } \\
\hline $\mathrm{H}_{2} \mathrm{CO}$ & $1_{11}-1_{10}$ & $4,829.7$ & $0.03 *$ & MPI 1 & $100-m$ & 2.6 & Schroder et al. (1974) \\
\hline $\mathrm{OH}$ & $1 / 2, F=1-0$ & $4,765.6$ & $0.03 *$ & $"$ & $"$ & $"$ & $"$ \\
\hline $\mathrm{OH}$ & $5 / 2, F=3-3$ & $8,189.6$ & 0.20 & NRAO & $140-\mathrm{ft}$ & 3.6 & Giguere and Clark (1975) \\
\hline $\mathrm{HC}_{3} \mathrm{~N}$ & $1-0, F=2-1$ & $9,098.3$ & 0.15 & $"$ & " & 3.2 & $"$ \\
\hline $\mathrm{HC}_{3} \mathrm{~N}\left(2 v_{7}\right)$ & $1-0, F=2-1$ & $9,156.1$ & 0.25 & $"$ & $"$ & $"$ & $"$ \\
\hline $\operatorname{HCN}\left(v_{2}\right)$ & $6, \ell$ doub. & $9,423.3$ & 0.40 & $"$ & $"$ & 3.1 & $"$ \\
\hline \multirow{3}{*}{$\mathrm{H}_{2} \mathrm{O}$} & ${ }^{6} 6^{-5_{23}}$ & $22,235.1$ & 1.07 & MPI 1 & $100-\mathrm{m}$ & 0.7 & Churchwell et al. (1975) \\
\hline & $"$ & $"$ & 0.02 & ARO 2 & $26-m$ & 1.4 & Avery and Andrew (1974) \\
\hline & $"$ & $"$ & $n r \star \star$ & NEROC & C 120 -ft & 1.5 & Jackson et al. (1975) \\
\hline $\mathrm{NH}_{3}$ & 3,2 & $22,834.2$ & 0.03 & ARO 4 & $46-m$ & 1.4 & Avery and Andrew (1974) \\
\hline $\mathrm{CH}_{2}(\mathrm{CN})_{2}$ & ${ }^{7} 16^{-7} 07$ & $23,084.2$ & 0.04 & ARO 4 & $46-m$ & $"$ & $"$ \\
\hline $\mathrm{NH}_{3}$ & 1,1 & $23,694.5$ & 0.7 & MPI 1 & $100-m$ & 0.7 & Churchwell et al. (1975) \\
\hline $\mathrm{CH}_{3} \mathrm{OH}$ & ${ }^{4} 2^{-4} 1$ & $24,933.5$ & 0.75 & $"$ & $"$ & $"$ & $"$ \\
\hline $\mathrm{CH}_{3} \mathrm{C}_{2} \mathrm{H}$ & $5_{0}^{-4} 0$ & $85,457.2$ & 0.4 & NRAO & $36-\mathrm{ft}$ & 1.3 & Buhl et al. (1975) \\
\hline$\left(\mathrm{CH}_{3}\right)_{2} \mathrm{O}$ & $2_{20}-211$ & $86,222.9$ & 0.4 & $"$ & $"$ & $"$ & $"$ \\
\hline $\operatorname{SiO}(V=1)$ & $2-1$ & $86,243.3$ & 0.1 & $"$ & $"$ & $"$ & $"$ \\
\hline HNCO & ${ }^{4} 04^{-3} 03$ & $87,925.2$ & 0.3 & $"$ & $"$ & $"$ & $"$ \\
\hline HNCO & ${ }^{4} 13^{-3} 12$ & $88,239.0$ & 0.4 & $"$ & $"$ & $"$ & $"$ \\
\hline $\operatorname{HCN}\left(2 v_{2}\right)$ & $1-0, F=2-1$ & $89,087.9$ & 0.3 & $"$ & $"$ & $"$ & $"$ \\
\hline $\mathrm{x}$-ogen $\left(\mathrm{HCO}^{+}\right.$ & +) 1-0 & $89,188.6$ & 0.3 & $"$ & $"$ & $"$ & $"$ \\
\hline "HNC" & $1-0$ & 90,665 & 0.3 & " & $"$ & $"$ & $"$ \\
\hline $\mathrm{HC}_{3} \mathrm{~N}$ & $10-9$ & 90,979 & 0.4 & $"$ & $"$ & $"$ & $"$ \\
\hline $\mathrm{CN}$ & $1-0$ & $113,491.0$ & 0.2 & NRAO & $36-f t$ & 1.1 & Ulich and Conklin (1974) \\
\hline$\infty$ & $1-0$ & $115,271.2$ & 0.5 & $"$ & $"$ & $"$ & $"$ \\
\hline
\end{tabular}


beamwidths witnout achieving detections in Comet Kohoutek. The well known small error in the topocentric ephemerides used by most radio observers has been discussed by several authors (e.g. Avery and Andrew 1974) and probably it had little effect on the outcome of most observations. However, ephemeris accuracy is most essential for compact sources and the high excitation required by the $6_{16}-5_{23} \mathrm{H}_{2} \mathrm{O}$ line suggests that in most comets it will be found only in a rather compact region. Thus, both the weak intensity of the $\mathrm{H}_{2} \mathrm{O}$ emission reported by Jackson et al. (1975) from Comet Bradfield and the generally low elevation of Comet Kohoutek suggest that atmospheric $\mathrm{H}_{2} \mathrm{O}$ attenuation coupled with a small pointing error may have been responsible for the negative $\mathrm{H}_{2} \mathrm{O}$ results found for Comet Kohoutek.

The upper limit for $\mathrm{H}_{2} \mathrm{CO}$ emission found by Schroder et al. (1974) for Comet Kohoutek was at least 100 times more sensitive than the upper limit found by Huebner and Snyder (1970) for Comet Bennett because of the narrower beam width used for the Comet Kohoutek search. Detection of the $1_{11}-1_{10}$ $\mathrm{H}_{2} \mathrm{CO}$ transition in comets may not be as straightforward as it appears, however, because if the excitation conditions are similar to the typical interstellar case, then the $\mathrm{H}_{2} \mathrm{CO}$ pumping mechanism may force tne absorption mode to predominate. The OH observations and radiative pumping calculations of Biraud et al (1974) suggest that preperihelion searches for $\mathrm{H}_{2} \mathrm{CO}$ absorption may be more successful than postperihelion searches in future comets

\section{SUMMARY}

We have learned that successful radio spectroscopy of comets almost always requires going to the detection limits of current instrumentation, which corresponds to a projected number density (in the initial energy level of the transition) of approximately $10^{12}$ molecules per $\mathrm{cm}^{2}$. Many of the 
radio observations appear to be consistent with a straightforward application of the fluid dynamic model (Huebner and Snyder 1970; Clark et al. 1971) with the possible exception of $\mathrm{H}_{2} \mathrm{O}$ (as discussed by Jackson et al. (1975)) and other molecules requiring unusual excitation for radio detection. Thus for most molecules of interest, reasonable estimates for the success of future searches can be obtained by starting from an expected production rate estimate, possibly based on the Comet Kohoutek results, and working backward to see if a projected density above $\sim 10^{12} \mathrm{~cm}^{-2}$ can be reached without excessive beam dilution.

Several of the successful radio detections have demonstrated that not all radio lines lead to a physically meaningful production rate due to the excitation conditions required for detection. Hence, as in the study of galactic molecular sources, non-LTE mechanisms will have to be introduced to explain the radio observations of both high lying transitions (e.g. $\mathrm{H}_{2} \mathrm{O}$ and vibrationally excited $\mathrm{CH}_{3} \mathrm{CN}$ ) and of easily inverted transitions (e.g. $\mathrm{OH}$ and passibly $\mathrm{H}_{2} \mathrm{CO}$ ). As a result, we can now start thinking about using future cometary observations to uniquely test several of the molecular pumping models which have been proposed for galactic molecular sources.

The unidentified lines found in Comet Kohoutek were at least as strong as the identified lines nearby in the spectrum and have not, as yet, been detected in the galactic molecular sources. This suggests that the radical and molecular ion chemistry of comets should be given more attention in the radio spectrum. In fact, it may be more interesting if radio astronomers in the future performed a few frequency scans on comets instead of limiting: themselves to grinding away on known transitions of stable molecules.

Finally, as a practical consideratjon, we have learned that both a good set of radio ephemerides and coordination among various groups of radio 
observers are essential to success It is doubtful that I would have as many cometary radio detections to discuss in this review had it not been for the efforts of Drs. 'T. A. Clark (NASA), D. K. Yeomans (Conp Sci Corp.), B. G. Marsden (SAO) and others in preparing and distributing useful ephemerides especially designed for radio observers The exceptional coordination between various radio observers and observatories optimized the use of valuable radio telescope time and was due mostly to the work of Drs. W. E. Howard (NRAO), S. P Maran (NASA), and R. W. Hoibs (NASA) Without tneir efforts, many of the radio molecular observations of Comet Kohoutek would not have occurred.

I wish to thanir the many radio observers who sent me their comet results for use in this review and acknowledge partial support for this work from NSF grant GP-34200 to the University of Virginia. 


\section{REFERENCES}

Avery, L. W., and Andrew, B. H. 1974, Astr.J., 79, 1322.

Biraud, F., Bourgois, G., Crovisier, J., Fillit, R., Gérard, E., and Kazes, I. 1974, Astr. and Ap., 34, 163.

Black, J. H., Chaisson, E. J., Ball, J. A., Penfield, H., and Lilley, A. E. 1974, Ap. J. (Letters), 191, LA5.

Buhl, D., Huebner, W. F., and Snyder, L. E. 1975, this publication.

Buh1, D., Snyder, L. E., Lovas, F. J., and Johnson, D. R. 1974, Ap. J. (Letters), $192, \mathrm{~L} 97$.

Churchwell, E., Landecker, T., Winnewisser, G., Hills, R., and Rahe, J. 1975 These proceedings, p. 297.

Clark, T A., Donn, B., Jackson, W. M., Sullivan, W. T. III, and Vandenberg, N. 1971, Astr. J., 76, 614 .

Davis, J. H., Blair, G. N., Van Till, H., and Thaddeus, P. 1974, Ap. J. (Letters), 190, Lll7.

Giguere, P. T. and Clark, F. O. 1975, Ap. J. (in press for 15 June).

Huebner, W F. 1970, Los Alamos Report LA-4542-MS.

Huebner, W. F., and Snyder, L. E. 1970, Astr. J., 75, 759.

Huebner, W. F., Snyder L. E., and Buhl, D. 1974, Icarus, 23, 580.

Jackson, W. M., Clark, T., and Donn, B. 1975, this publication.

Potter, A. E., and Del Duca, B. 1964, Icarus, 3, 103.

Schröder, R., Wendker, H. J., and Stumpff, P., 1974, Astr. and Ap. 37, 417.

Snyder, L. E., and BuhI, D. 1974, Ap. J. (Letters), 189, L31.

Snyder, L. E., Huebner, W. F., and Buhl, D. 1975, Comet Kohoutek Workshop,

NASA/MSFC, (NASA Special Publication, in press).

Thaddeus, P., Mather, J., Davis, J. H., and Blair, G. N. 1974, Ap. J. (Letters), 192, L33.

Turner, B. E. 1974, Ap. J. (Letters), 189, L137.

Ulich, B. L., and Conklin, E. K. 1974, Nature, 248, 121.

Watson, J K. G. 1974 (preprint). 


\section{DISCUSSION}

H. Keller: I would like to ask if you would define a flare, and also do you have any idea what the scale length of $\mathrm{H}_{2} \mathrm{O}$ was?

L. Snyder: Okay. Your second question, I think should be directed to Jackson, who's sitting here.

The first question - I can define a flare as anything that excites a vibrationally excited state. In this case we think it may have been a knot that started passing from the head of the comet to the tail about the 1st of December. But you can take the usual definition of flare and see what it does in the radio spectrum, or you can take the radio definition and then say if this excitation is unusual, it's certainly flaring in the radio.

L. Biermann: Could you say anything concerning the upper limit for the production rate of $\mathrm{CO}$ given by Conklin and Ulich? It's dipole moment is very small.

L. Snyder: They gave upper limits for CN and CO. First of all, I'd just like to say a word about the upper limits. Given the current state of telescope technology and receiver technology, if you can't see a molecule, the upper limit on the projected density for the state of interest usually comes out to be about $10^{11}$ or $10^{12}$, This in turn, given reasonable cometary dimensions and a ground-state rotational transition, will invariably lead to a production rate on the order of $10^{26}$ or $10^{27}$. But what I think happened in the $\mathrm{CN}$ upper limit, was that they got a beautiful spectrum of vibrationally excited $\mathrm{CH}_{3} \mathrm{CN}$, two $\mathrm{K}$ components. And if I were to guess, I would say that if one could remove the methylcyanide lines, one might see a velocity broadened $\mathrm{CN}$ line.

\section{Biermann: What about CO?}

L. Snyder: A production rate less than $10^{29}$ would be consistent with the CO negative results.

Voice: Is that a ground state transition?

L. Snyder: Yes. 1-0.

F. L. Whipple: I want to congratulate Dr. Snyder and all the workers here, but I wanted to remove one misapprehension, at least in my opinion. I think that's off the record that we consider cometary astronomy in a very bad state.

(Laughter.) 


\section{DISCUSSION (Continued)}

L. Snyder: You mean it's come full circle.

F. L. Whipple: It hasn't gotten very far yet.

\section{(Laughter.)}

W. Jackson: I wanted to make a few comments about the radio observations. One is that the comet Bradfield production rates, as we realized, are completely ridiculous, in terms of the water production rate. I think that it points up the fact that what we may be seeing is a reflection of the excitation mechanism. Even the production rate for methylcyanide as calculated originally by Ulich and Conklin, and I think that's the original number, is too low if you use reasonable values of the radius of the comet - I mean I think they use something like 10 kilometers or 20 kilometers for the radius of comet Kohoutek, and I think something more reasonable is like 5 . The signal goes as the square of the radius of the nucleus, so even that suggests that indeed methylcyanide must be produced either in a flare or in a nonequilibrium mechanism, as the vibrational excitation indicates.

The beam dilution, it turns out, is really a function of the lifetime of the cometary species; the beam dilution in formaldehyde is greater than the beam dilution in water, or it should be.

L. Snyder: It's also a function of wavelength and telescope diameter, so if you put the proper configuration -

W. Jackson: Yeah. Okay, okay, I agree. - But I'm just saying the overall cloud size is going to be dependent upon the photodissociation rate. And it turns out for water the photodissociation rate ought to be almost a factor of 4 lower than the photodissociation rate of formaldehyde.

H. Keller: What is the photodissociation rate for $\mathrm{H}_{2} \mathrm{O}$ ?

W. Jackson: The lifetime I get is something like 2 times $10^{4}$ seconds. And for formaldehyde it is 5 or 6 times $10^{3}$ seconds.

H. Keller: At one AU?

W. Jackson: One AU. Everything I say is referred to $1 \mathrm{AU}$.

I've puzzled over why we saw water in Bradfield rather than water in Kohoutek. If I may go to the board for a minute, I think part of it may be due to the way we were pointing in Bradfield. 


\section{DISCUSSION (Continued)}

I don't know much - I'm not really a radio astronomer, but I was up there for both of the observations, and in Kohoutek, if this is the earth -

We were pointing the telescope at low elevation through a large atmospheric mass. The comet was down close to the horizon all the time.

Bradfield was much higher - near the pole, and was circling around the pole. Now, the noise in the case of Kohoutek increases measurably compared to the noise for Bradfield.

The effectiveness of the integration is much better for Bradfield because even though we may have integrated for 4 or 5 hours on Kohoutek most of it was under conditions where I would say that the signal to noise ratio was poorer than under the 4 or 5 hours for Kohoutek.

L. Snyder: Okay. If you combine this, a $\tau$ secant $\mathrm{Z}$ for the atmosphere correction, say, with even a quarter of a beam width mispointing, the signal could disappear in the noise very easily. I'd just like to add a little bit to that, and I think that the nonequilibrium detections - methylcyanide, water vapor, HCN I think are going to prove to be very important for understanding what happens in the interstellar clouds. Because if you consider for a minute - if you get enough telescope time and enough observational time, as the French did on the $\mathrm{OH}$, as you track the comet, and as you can see signal variations with heliocentric distance, it gives you very direct measures, then, on what the UV pumping, for example, is doing to a particular molecule. You just can't do it in the interstellar medium.

I think it's a very promising area of future research, which has come out of this.

G. Herzberg: May I ask to what extent you guard - you have to guard against accidental overlapping of some background source. I mean your comet, while you are averaging for several hours, goes over a lot of the sky. Now, there may by chance be -

L. Snyder: That's right. That's right.

Now, the primary way we guard against this is by looking at each scan as it comes in, before we do any stacking, do very careful editing. The secondary way we guard against it is to make sure that we are clear of the known sources, and then a third way, if any detection is suspect, the common way is just to go back and point in that direction after the comet's gone by. And if there's a source there, then the signal will come in even though the comet's gone. 


\section{DISCUSSION (Continued)}

E. Ney: Maybe this is crazy, but what makes infrared so easy is that you can find a comet in the daytime, peak up on the signal, get the telescope tracking, and then go integrate on hard wavelengths.

What are the chances that you'll find something bright in the radio to track on? For example, broad band thermal emission from the dust?

L. Snyder: There were several thermal emission experiments tried at various frequencies, and I think the only one that was successful was the interferometer experiment of Hobbs and Maran and Webster and - who else was on it - at Greenbank. And when I talked to Bob Hobbs a few minutes ago, they were still squabbling over the results, because again, it's a very, very weak signal. It's less than 70 flux units, Bob says.

E. Ney: It's just not bright.

L. Snyder: There's nothing bright, given present receivers. Now, if we can cut receiver noise maybe in half, maybe the time will come when we could do that. But given the beam dilution and given the receivers, it's a pretty impossible job. We can't even map now, given our beams and our signal to noise.

We'd like to reach the place where we could map on comets, too, which is a sensitivity problem.

So as far as I know - Dave, did you have something to add to that?

D. Buhl: There was - I believe it was the French group detected the comet at 1 millimeter in the continuum. If the receivers are improved considerably, 1 millimeter might be the frequency for that type of thing.

P. Wehinger: In regard to the background noise that you were commenting on, in the case of Bradfield, it was going through the galactic plane as a background noise. Are there any problems with this compared to the case where Kohoutek was well out of the galactic plane?

L. Snyder: Why don't you ask the man who owns one, sitting in front of you? Jackson did the Bradfield observations.

W. Jackson: Well, the way the radio observations are done, we are looking first where we expect the comet to be 10 minutes later. And then we're looking where the comet is now. So if it was in a galactic noise source, the background signal measured ten minutes earlier would have 


\section{DISCUSSION (Continued)}

included the galactic source also. Subtracting the background would eliminate the galactic source.

L. Snyder: All right. You're beam switching there?

W. Jackson: Yes.

B. G. Marsden: I'd just like to distinguish between errors in the ephemeris and errors in the use of the ephemeris.

(Laughter.)

How accurately do you actually want the ephemeris?

L. Snyder: Well, okay. I brought along some numbers. It seems to me that if we're going to probe the nucleus of the comet, we want to probe at millimeter wavelengths.

This means that our largest beam width will be on the order of 80 seconds of arc, and our smallest one, for all practical purposes, given today's telescopes, will be about 40 seconds of arc. So we don't want anything probably more accurate than 10 seconds of arc, because that's the pointing accuracy limit, but if you could take it down to the pointing accuracy -

B. G. Marsden: Down to 30 seconds, say -

L. Snyder: Well, 10 seconds would be great -

B. G. Marsden: Then down to 10 seconds.

L. Snyder: Right.

B. G. Marsden: The custom is, of course, that ephemerides are published by combining the geocentric coordinates of the earth for universal midnight, or ephemeris midnight, with the heliocentric coordinates of the comet for that time. Now, this is absolutely useless, because nobody ever observes that, but this is the convention. What you really want, I suppose, is for us to antedate the heliocentric coordinates of the comet by the light-time, and produce an astrometric ephemeris.

But even then you'd be getting into trouble with parallax, if you aren't already when you're getting down to it at ten seconds. But this is the convention 


\section{DISCUSSION (Continued)}

of the IAU; I suppose we have to ask Commission 20 if it will change its convention.

L. Snyder: Yes, well, times are changing in radio, so I think it would be a fair request, because as Jackson points out, when you have a low-lying comet, and you're trying to do water, and in addition you have a half a beam width error, as this pair of slides showed, it can just take the signal right down into the noise. And in fact, there was some hint of a signal on water in Kohoutek; but it really couldn't be proven, couldn't be verified above the noise.

Isn't that correct?

W. Jackson: Yes, There are some measurements that hint that there might have been some water present. But not enough that you could look at it and say with confidence, especially since I'm not a radio astronomer.

L. Snyder: And this optical checking business probably isn't any better than a beam width where it's tracked with the telescope mounted beside the radio telescope, because the axes don't exactly coincide.

B. G. Marsden: Well, we have provided in a few cases on request, specialized ephemerides for certain people, actually for the location of their radio telescope. How useful is it to do this after the event?

L. Snyder: Then it's usually too late. Except for the cases where we have a strong signal of an unidentified feature and we want to get the rest frequency on it and determine whether it's ethyl alcohol or acetone or what have you.

B. G. Marsden: Did you have ethyl alcohol, perhaps?

L. Snyder: I have an announcement from the Washington Post here that I could read, but I won't.

(Laughter.)

W. Jackson: I'd like to make one more comment about the radio measurements in the nonequilibrium excitation mechanism. If you examine the infrared measurements of Meisel as I originally saw them - and I'm quoting from a preprint - the original $\mathrm{CN}$ to $\mathrm{OH}$ concentration was such - by the infrared measurements - you would predict that there was more $\mathrm{CN}$ in the comet than $\mathrm{OH}$. 


\section{DISCUSSION (Continued)}

And this depended, as I understood the analysis, on a thermal mechanism for the production of the $\mathrm{CN}$ infrared emission. That contradicts the UV measurements as we know them, of $\mathrm{CN}$ and $\mathrm{OH}$. We understand the excitation mechanism of the $\mathrm{CN}$ and $\mathrm{OH}$ emission in the UV region.

All I'm trying to say, really, is that one of the exciting things about the possibilities of observing comets in the infrared and radio regions, it may lead to more information about the chemistry of comets, since you are measuring transistions that may be excited by new mechanisms.

M. Mumma: I'd like to say a few words on that, which bear also on the question of the accuracy of the ephemerides. This winter a group of people here at Goddard, and also at NRAO, including Dave Buhl, myself, Ted Kostuk Steve Cohen and Peter von Thuna, from Arthur D. Isittle, built a new instrument called an infrared heterodyne spectrometer, which promises the ability to measure IR emissions from parent molecules in the inner coma, with 5 arc second resolution in the neighborhood of 10 microns.

This is obviously going to be an important new tool for study cometary processes, and it's one that is now available.

So I would say to Brian that we could use ephemerides that reflect the pointing accuracy that we can now achieve with this instrument.

Voice: I hate to sound skeptical, but I just wonder what criterion you use for the identifications you have in the microwave spectra. For example, can you tell me how many random noise spectra you would feed in for each identified spectrum? In other words, is the chance 1 in 10, 1 in 20 of this being spurious?

L. Snyder: In our own particular data, I think that the unidentified spectra stand alone, in that we have significantly no scans in which they're not showing up above the noise level. So I don't think we have a statistical problem there.

In the HCN data that we report, our common practice is to break the scans into groups, stack the groups and see if we can still see the line, and then try mixing the groups from different days, and see if we can still see the line. In the case of the central features in the $\mathrm{HCN}$, which are all above the noise, we can.

Now, in the case of the methylcyanide data (both the $V_{8}=1$ state and the ground state data) if the features that were appearing at about 2 to 1 or 2.5 to 1 did not match the velocity which had been reported, say, a couple weeks earlier with about 4 to 1 signal to noise by Ulich and Conklin, then we couldn't even 


\section{DISCUSSION (Continued)}

report them. I show them here as a confirmation of their detection, but they do not qualify as independent of their detections.

But I don't really think that's the point in identifying a radio line. Or if it is, it doesn't explain enough. In order to identify a radio line, first of all, as we've discussed earlier, you have to make sure the pointing is on the comet and not on some galactic object. And then you have to go through a series of local oscillator shifts, and see if the line moves as you shift the local oscillator. If it moves in your filters or your autocorrelator, because you're moving the box around an absolute spectrum, so as you shift one way the line should move one way, and as you shift the other way the line should move the other. Any real feature that's coming in, say, from the comet or a galactic source, will move, and any noise feature will not move. And when you build up signal to noise on a feature that moves several times, The statistical argument, then, boils down to what the proper intensity is in terms of signal noise.

E. Ney: I'm afraid this may even be almost insulting, but I assume that you handled differential refraction. You have to have your ephemeris, of course, but you have to also put in refraction.

L. Snyder: Well, we take that out with our pointing.

That's integral to our pointing procedure.

E. Ney: But differential refraction, as you track down toward the horizon what do you do about that?

L. Snyder: Well, as we track down toward the horizon, we try to correct our pointing accordingly.

E. Ney: Toward the differential refraction?

L. Snyder: We know where we're trying to point, and we know where our objects are that we have as standard check objects. I think that question has come up before on Bennett and I think that's the best answer I can give you for now.

E. Ney: But it's big. You go another air mass and it's another minute of arc, so if you're pointing at an ephemeris position you'll be out of the beam.

D. Buhl: (inaudible) 
L. Snyder: What Dave Buhl said was that it's in the telescope pointing program, and it's a problem that you have with any kind of source. It's essentially taken care of in a series of pointing sections every few months and it's programmed in.

Does that answer your question? Okay.

Because - well, yes, that's a good point. We track galactic sources over the horizon, for example, quite commonly, and I must say that the horizon pointing, which takes into account differential refraction, sometimes is much better than the zenith pointing. We sometimes get better signal to noise as the source is going over the horizon than we do at zenith on the 36-foot telescope.

So I'm happy that the corrections are proper from the data.that I've seen in the past.

W. F. Huebner: 'Two models are being proposed for the formation of comets. One of them deals with the formation in the solar nebula. The other one, in a companion nebula, the composition of the comet would therefore be different in both of them.

If the formation of the comet were to occur in the solar nebula, you would expect chemistry which is more like equilibrium. I put the emphasis on "more like," not on "equilibrium."

Can you tell us what the ratios are of the "exotic molecules" to $\mathrm{H}_{2} \mathrm{O}$ in interstellar space? Because I think that would be an important criterion to differentiate between the two models.

L. Snyder: Okay, I can give you some numbers. But one thing to keep in mind, which I pointed out before, that given the models, probably the only molecule that we have going here that'll give you a somewhere near legitimate production rate, is one that involves low-lying transitions in the ground-rotational state, and that's HCN at this point.

So in the future we can choose our molecules particularly for production rate calculations, it would seem to me. In terms of comparing the interstellar medium to the comet, of course we get varying numbers as we go around the interstellar medium, but if we pick the Orion cloud, for example, and try to compare projected densities along a given length, we have to say that the molecules along a given length for the heart of the Orion nebula is probably about $10^{+15}$ integrated column density per centimeter squared.

Now, when we try to compare that with water, we run into exactly the same problem we're running into in the comet, and that is that the water is masering, 


\section{DISCUSSION (Continued)}

it's time-varying, and in Orion it has about a dozen velocity components. So you cannot use a direct measurement of water in Orion, but you can say, "If I look around at the other polyatomic molecules that are seen in Orion, and I look at the diatomic molecules that are seen in Orion, quite typically for $\mathrm{CO}$, which is very bright in Orion - it's about $10^{18}$ per centimeter squared.

And $\mathrm{OH}$, from what can be deduced from absorption measurements, is about $10^{17}$ per centimeter squared. $\mathrm{HCN}$ is about $10^{15}$, and then when we drop down to isocyanic acid and molecules of this complexity then we're talking about $10^{12}$, approximately, per centimeter squared. I'm a little hesitant on this number, because we just finished this detection. I haven't really completed all the analysis on it.

So let me raise this to $10^{13}$, and again, dimethyl ether - in Orion, again is about $10^{13}$ per centimeter squared.

Okay. So if you bootleg it like that, and say - I don't know of any peculiarity in the interstellar chemistry, given enough hydrogen and given enough oxygen, that would cause water to deviate very much from this scheme - then $I$ think that probably within an order of magnitude either way, water should be about $10^{16}$, if you could see thermal water in Orion. You can't, so we don't have a better number than that, and if you wish, for your purposes you can choose $10^{15}$, but I don't think you can choose $10^{18}$ at this point.

B. Donn: This is a point I was going to discuss in my paper, on Wednesday, on the origin of comets. It seems to me this is an important way of looking at the origin. One can, in a sense, divide the composition of comets, including new comets, into a number of different classes, depending on the ratios $\mathrm{CO}^{+}$and $\mathrm{N}_{2}^{+}: \mathrm{CN}, \mathrm{C}_{2}, \mathrm{C}_{3}, \mathrm{NH}$, etc: continuum and try to fit this into some formation pattern which I will do on Wednesday. 